\title{
Bone marrow-derived mononuclear cell therapy induces distal angiogenesis after local injection in critical leg ischemia
}

Jean-Paul Duong Van Huyen ${ }^{1,2}$, David M Smadja ${ }^{1,3}$, Patrick Bruneval ${ }^{1,2}$, Pascale Gaussem,3, Liliane Dal-Cortivo ${ }^{1,4,5}$, Pierre Julia ${ }^{1,6}$, Jean-Noël Fiessinger ${ }^{1,7}$, Marina Cavazzana-Calvo $^{1,4,5}$, Martine Aiach $^{1,3}$ and Joseph Emmerich ${ }^{1,7}$

${ }^{1}$ University Paris Descartes, Paris, France; ${ }^{2}$ INSERM UMRS 872 and Department of Pathology, Hôpital Européen G. Pompidou, Paris, France; ${ }^{3}$ INSERM U765 and Hematology-Haemostasis laboratory, Hôpital Européen G. Pompidou, Paris, France; ${ }^{4}$ Département de Biothérapies, Hôpital Necker-Enfants Malades, Paris, France; ${ }^{5}$ INSERM U 768, Paris, France; ${ }^{6}$ Department of Cardiac and Vascular Surgery, Hôpital Européen G. Pompidou, Paris, France and ${ }^{7}$ INSERM U765 and Department of Vascular Medicine and Hypertension, Hôpital Européen G. Pompidou, Paris, France

Critical leg ischemia is associated with a high risk of amputation when revascularization is not possible. Cell therapy based on bone marrow-derived mononuclear cells or with peripheral mononuclear cells, collected after stimulation with G-CSF has been used in an attempt to stimulate angiogenesis. Although several studies have raised the hope that such cell therapy may be effective in critical leg ischemia, no direct demonstration of angiogenesis induced by bone marrow-derived mononuclear cell/peripheral mononuclear cell injection has been reported in man. The aim of this study was to identify and to evaluate the extent of the angiogenic process associated with cell therapy in critical leg ischemia in man. To address this question, this pathological study was conducted in patients enrolled in the OPTIPEC clinical trial (Optimization of Progenitor Endothelial Cells in the Treatment of Critical leg ischemia), an interventional cell therapy study in critical leg ischemia. Amputation specimens from these patients were submitted to a standardized dissection protocol. In three patients, an active angiogenesis was observed in the distal part of the ischemic limb but not in the gastrocnemius muscle, the site of bone marrow cell injection. All the newly formed vessels were positive for endothelial cell markers (CD31, CD34, von Willebrand factor) and negative for markers of lymphatic vessels (podoplanin). Immunohistochemical staining for Ki-67 and c-kit showed extensive endothelial cell proliferation within the new vessels. Bone marrow-derived mononuclear cell therapy in patients with critical leg ischemia induces an active, substained angiogenesis in the ischemic and distal parts of the treated limb, although this may not prevent amputation in some patients with very severe ischemia.

Modern Pathology (2008) 21, 837-846; doi:10.1038/modpathol.2008.48; published online 16 May 2008

Keywords: angiogenesis; bone marrow mononuclear cells; cell therapy; ischemia; peripheral artery disease

Critical limb ischemia related to atherosclerosis is associated with a high risk of amputation and death. Despite improvements in the medical and surgical treatment of critical limb ischemia, $10-40 \%$ of patients still require amputation. ${ }^{1}$ The risk is even higher in patients who are not amenable to surgical revascularization or angioplasty owing to the absence of distal run-off. Several medical options have

Correspondence: Dr J-PD Van Huyen, MD, PhD, INSERM UMRS 872 and Service d'Anatomie Pathologique, 20 rue Leblanc, Paris Cedex 15 75908, France.

E-mails: jp.dvh@wanadoo.fr and

jean-paul.duong-van-huyen@egp.aphp.fr

Received 17 September 2007; revised 04 February 2008; accepted 10 February 2008; published online 16 May 2008 been tested in patients who cannot be revascularized, but none has shown long-term efficacy. ${ }^{1-3}$

Occlusion of the arterial tree causes tissue hypoxia, which is a strong stimulus for angiogenesis. ${ }^{4,5}$ The development of collateral vessels occurs physiologically in patients with critical limb ischemia and is mainly driven by an increased endogenous angiogenic response. ${ }^{6}$ This process is responsible for increased microvessel density in muscles of patients with critical limb ischemia, partially compensating for the occlusion of native arteries but failing to restore normal flow. Various attempts have been made to augment this angiogenic process in patients with ischemic disease. ${ }^{3,7,8}$

Cell-based therapy is a novel and attractive potential treatment strategy for patients with critical 
limb ischemia, based on the fact that endothelial cells and hematopoietic stem cells derive from a common precursor, the hemangioblast. Endothelial progenitor cells derived from bone marrow circulate in peripheral blood and are involved in regenerating injured endothelium and in neoangiogenesis after tissue ischemia (review in Losordo and Dimmeler ${ }^{7,8}$ ). These cells were first identified by Asahara, who demonstrated their ability to contribute to new vessel formation. ${ }^{9}$ The origin of endothelial progenitor cells involved in the angiogenesis that follows vascular damage or ischemia is still a matter of debate, and two types of cells have been used in preclinical trials: early endothelial progenitor cells of monocytic origin $\left(\mathrm{CD} 14^{+}\right)$and late or outgrowth endothelial cells $\left(\mathrm{CD} 14^{-}\right) .{ }^{10}$ These cells, of CD34+ origin, can differentiate into endothelial cells, as shown by their expression of various endothelial markers (KDR, von Willebrand factor, VE-cadherin, CD31). In animal models of hind-limb ischemia, injection of endothelial progenitor cells markedly improved blood flow and capillary density. ${ }^{8}$ Both early and late endothelial progenitors can induce angiogenesis in animal models, and a synergistic effect is obtained by mixing these two cell types. ${ }^{11}$

While different cell types of distinct origins can induce angiogenesis in vivo in animal models, only bone marrow mononuclear cells and peripheral blood mononuclear cells, collected after mobilization with granulocyte colony-stimulating factor (G-CSF), have been used in clinical trials. ${ }^{12-16}$ The first human trial of cell therapy in critical limb ischemia was published in 2002 by Tateishi-Yuyama et $a l,{ }^{12}$ who investigated the efficacy and safety of autologous bone marrow mononuclear cells. Huang et $a l^{15,16}$ reported a slightly different approach based on G-CSF-mobilized peripheral blood mononuclear cells. Although the positive clinical results obtained by these studies clearly bring new insight in the safety/ efficacy of cell therapy in critical limb ischemia, no direct demonstration of angiogenesis induced by bone marrow mononuclear cell/peripheral blood mononuclear cell injection has been reported in man.

The aim of this study was to identify and to evaluate the extent of the angiogenic process associated with cell therapy in critical limb ischemia in man. To address this question, this study was conducted in patients enrolled in the OPTIPEC clinical trial (Optimization of Progenitor Endothelial Cells in the Treatment of Critical limb ischemia), an interventional cell therapy study conducted in our institution. We thus systematically performed pathological examination of lower limbs in study cases undergoing amputation.

\section{Materials and methods}

\section{Patients}

The four subjects of this study were enrolled in the OPTIPEC clinical trial. The OPTIPEC clinical trial is a multicentric, phase I, safety/efficacy, interventional, non-randomized study with a single group assignment. The protocol was approved by the Paris-Broussais-HEGP ethics committee and all the patients gave their written informed consent. Patients were eligible if they had critical limb ischemia associated with limited gangrene or nonhealing ischemic ulcers and if they were not eligible for surgical revascularization or for percutaneous angioplasty, or if such procedures had little chance of success. On the basis of the TASC consensus and current guidelines, ankle pressure had to be below $70 \mathrm{mmHg}{ }^{1}$

The cell therapy protocol was similar to that initially published by Tateishi-Yuyama et al. ${ }^{12}$ Briefly, under general anesthesia, $500 \mathrm{ml}$ of bone marrow was aspirated from both posterior iliac crests. Mononuclear cells were immediately concentrated to a final volume of $30 \mathrm{ml}$. A buffy coat was first prepared to reduce the volume and to remove red cells. Mononuclear cells were then isolated on a Cobe 2991 device by centrifugation on a Ficoll density gradient, as described previously. ${ }^{17,18}$ After washes, bone marrow mononuclear cells were suspended in $30 \mathrm{ml}$ of $4 \%$ human serum albumin solution (LFB, Les Ulis, France). The initial and final products were subjected to total and CD34 + cell counts and sterility controls. Less than $3 \mathrm{~h}$ after cell isolation, 40 injections of $0.75 \mathrm{ml}$ each were made in the gastrocnemius muscle of the ischemic limb. All the patients were examined each week during the first month, and then every month for 1 year.

Four patients with critical limb ischemia, age- and sex-matched, who were not included in the cell therapy protocol and who have been amputated during the same period were analyzed as controls.

\section{Tissue Sampling}

All the amputation specimens were submitted to a standardized dissection protocol. When the limb was amputated below the knee, a large sample of gastrocnemius was sampled, around the site of cell injection and along the tibial arteries. The forefoot tissues and arteries (plantar and toe arteries) were also sampled in each case. Between 20 and 25 paraffin blocks were prepared in each case and were stained with hematoxylin and eosin (H\&E).

\section{Immunohistochemistry}

On the basis of H\&E staining, angiogenesis was defined as a focal or diffuse area of hypervascularization with new vessels remarkably observed in unusual localization such as within aponeurosis, adipose tissue and media of the vessels. Tissue samples showing angiogenesis were selected and submitted to immunohistochemistry. The following markers were used: anti-CD31, anti-CD34, and 
anti-von Willebrand factor (all from Dako, Trappes, France) as endothelial cell markers; anti-podoplanin (Bender MedSystems, Vienna, Austria) as a lymphatic endothelial cell marker; anti- $\alpha$-smooth muscle actin (Dako) as a vascular smooth muscle cell marker; anti-Ki67 antibody (Dako) as a proliferation cell marker; anti-CD117 (c-kit; Dako) as a precursor endothelial cell marker; anti-Glut-1 (Dako), expressed in endothelial cells of some angiomas and angiosarcomas. Immunohistochemical studies of paraffin sections were performed following standard procedures, using a three-step avidin-biotin immunoperoxidase method. ${ }^{19}$

\section{Quantification of Angiogenesis}

The quantification of angiogenesis was performed by determining the microvessel density in the angiogenesis areas and in the areas devoid of angiogenesis in the patient specimens and within the amputation specimens of the paired controls. The microvessel density counts were performed using a 25 dots Chalkley graticule (grid area: $0.196 \mathrm{~mm}^{2}$ ) as described previously. ${ }^{20}$ The Chalkley count for each area was the mean of five counts on CD34 stained sections.

The extent of the angiogenesis process within the amputation specimens was then determined, first by counting the number of blocks with area of angiogenesis and second by measuring the area with angiogenesis in the tissue sections. All H\&E stained sections were numerized (ICS capture software, Tribvn, France) and a computer-assisted analysis of the angiogenesis area was performed (ICS framework viewer, Tribvn, France).

\section{Results}

\section{Clinical Findings}

The characteristics of the four patients treated with bone marrow mononuclear cells are shown in Table 1 . The mean age of the patients, all of whom were men, was 69 years (range 54-88). The first patient was enrolled in March 2005 and the last in April 2006. One of the patients had non-insulindependent diabetes mellitus for more than 10 years, and three had a history of heavy smoking. All the patients were evaluated for distal run-off by selective arteriography less than 3 months before enrollment in the protocol, and were found to have unreconstructible critical limb ischemia. The bone marrow harvesting procedure was well tolerated. The mean total bone marrow mononuclear cell count obtained after concentration to a volume of $30 \mathrm{ml}$ was $2.61 \times 10^{9}$ (range $\left.1.11-4.49 \times 10^{9}\right)$. The mean number of $\mathrm{CD} 34+$ cells reinjected was $69.25 \times 10^{6}$, corresponding to a mean of $0.95 \times 10^{6}$ cells $/ \mathrm{kg}$ (range $0.39-1.93 \times 10^{6}$ ). The cells were then reimplantated by $30-40$ intramuscular

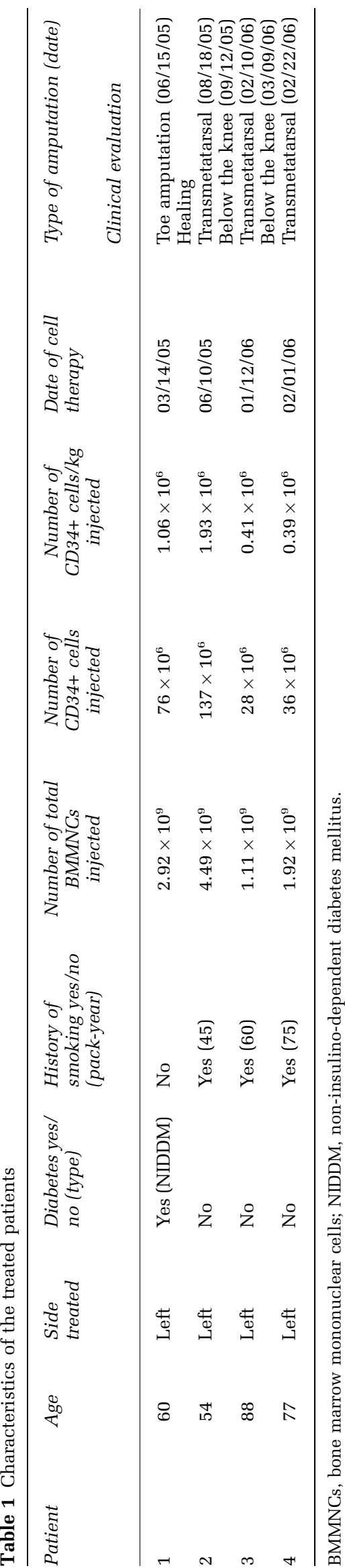


injections without any local complications, including hematomas.

Clinically, the local outcome of critical limb ischemia was favorable in one patient (case 1), with a limited toe amputation followed by healing of the amputation scar. The other three patients required transmetatarsal amputation, followed by below-theknee amputation in two cases owing to the progression of gangrene. All patients were amputated in our department.

\section{Pathology}

The pathological findings in the patients are shown in Figures 1-3. The quantification of the extent of angiogenesis is given in Tables 2 and 3 .

\section{Patient 1}

In Patient 1, the toe amputation was devoid of angiogenic process in this limited amputation specimen.

\section{Patient 2}

In this patient, the patient's below-the-knee amputation specimen, gangrene extended beyond the first metatarsal. Chronic thrombosis and severe atherosclerosis and arteriosclerosis were observed on arterial sections. No changes were observed in the gastrocnemius muscle. Distal tissues of the forefoot and base of the toes showed extensive angiogenesis (Figure 1, left panel), consisting of large hypervascularized foci in soft tissues and vessel walls. In some areas, the angiogenesis appeared more aggressive, with invasion of the fascia (Figure 1). Soft tissues were colonized by small vessels, capillaries and venules comprising a layer of vascular smooth muscle cells (Figure 2). Medium-sized arteries and veins showed marked angiogenesis in their walls, whereas their lumens were patent (Figure 2). All the newly formed vessels were outlined by a continuous endothelium expressing the endothelial cell markers CD31, CD34 and von Willebrand factor (Figure 1), and negative for the lymphatic endothelial cell marker podoplanin (data not shown). Many endothelial cells expressed the stem cell marker c-kit (Figure 3). These cells retained a high proliferative potential, as $5 \%$ were positive for Ki67, whereas endothelial cells in proximal vessels remote from foci of angiogenesis were not labeled. The latter cells did not express Glut-1 (data not shown).
Gastrocnemius muscle specimens contained no hypervascularized areas.

\section{Patient 3}

In this patient, the patient's forefoot amputation specimen, the toes were gangrenous. Chronic thrombosis and moderate arteriosclerosis were observed on arterial sections. In the forefoot, limited foci of angiogenesis (Figure 1, middle panel) were observed in soft tissues and vein walls, remote from the areas of gangrene. They were composed of venules with a vascular smooth muscle cell layer (Figure 2). They expressed endothelial markers (Figure 1), but not the lymphatic endothelial marker podoplanin or the stem cell marker c-kit. The endothelial cells were proliferating and frequently expressed Ki67 (Figure 3).

\section{Patient 4}

In this patient, the patient's forefoot amputation specimen, the toes were gangrenous. Severe arteriosclerosis with mediacalcosis and microangiopathy was observed. In the forefoot, rare foci of angiogenesis were observed in soft tissues and vein walls, consisting mainly of venules (Figure 1, right panel). The endothelial cells expressed endothelial markers (Figure 1) but not the lymphatic endothelial marker podoplanin. Some cells expressed c-kit (Figure 3). They were proliferating and frequently expressed Ki67. The gastrocnemius muscle sample did not contain hypervascularized areas.

\section{Controls}

Four age- and sex-matched patients (mean age 68, range 44-83) with critical limb ischemia who had major amputation during the same period had pathology examination using exactly the same protocol. In control patient tissues, there was no focus of increased vascular density, reflecting the absence of angiogenesis (Figure 4). This was observed in all tissue samples including those from ischemic areas adjacent to gangrene. No angiogenesis was present in the vein and the artery walls. In the endothelial cells, c-kit expression and cell proliferation were not observed (Figure 4).

\section{Quantification of angiogenesis}

The microvessel density measurements showed that the Chalkley counts were threefold higher in the angiogenic areas than in the non-angiogenic

Figure 1 Main histological characteristics and expression patterns of endothelial cell markers in three patients. (H\&E stain and CD34, CD31, and von Willebrand factor immunohistochemistry. Scale bar $=100 \mu \mathrm{m}$ ). H\&E histology: In patient 2 , aggressive vascular proliferation disrupted a fibrous fascia (asterisk); in patient 3, angiogenesis was less extensive and had an angioma-like aspect (arrows); in patient 4, angiogenesis involved dilated or branching venules. Endothelial cell marker immunohistochemistry: Patient 2: CD34-panel clearly shows the prominent periarterial (a) and perivenous (v) distribution of the massive angiogenesis. In patient 2 CD31-panel, endothelial labeling confirmed that the fibrous fascia was disrupted by angiogenesis. In the three patients, all the newly formed vessels strongly expressed all the endothelial markers tested. 
Patient 2
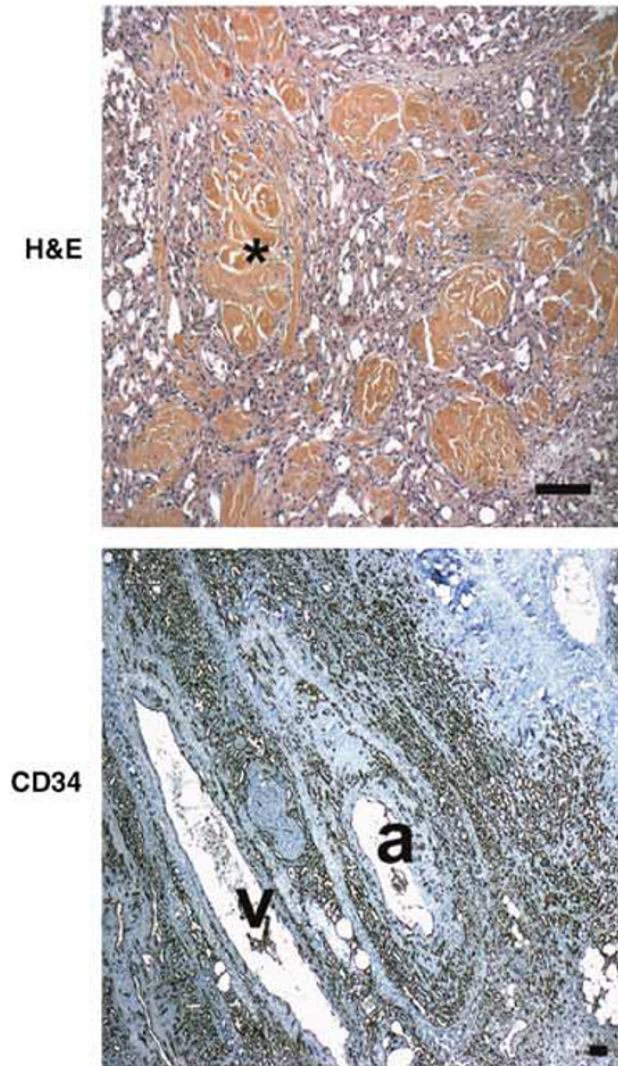

CD31
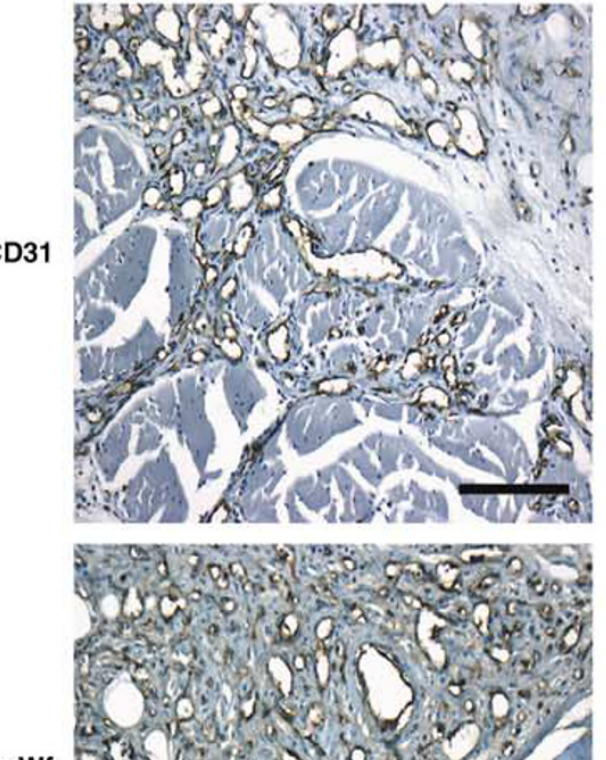

vWf

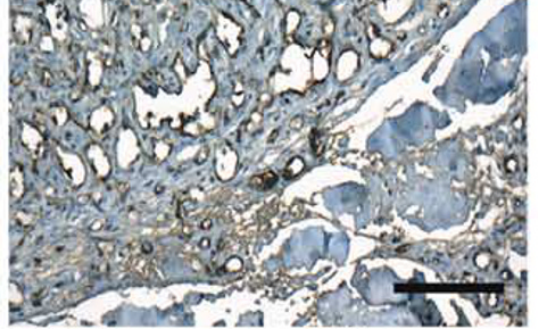

Patient 3
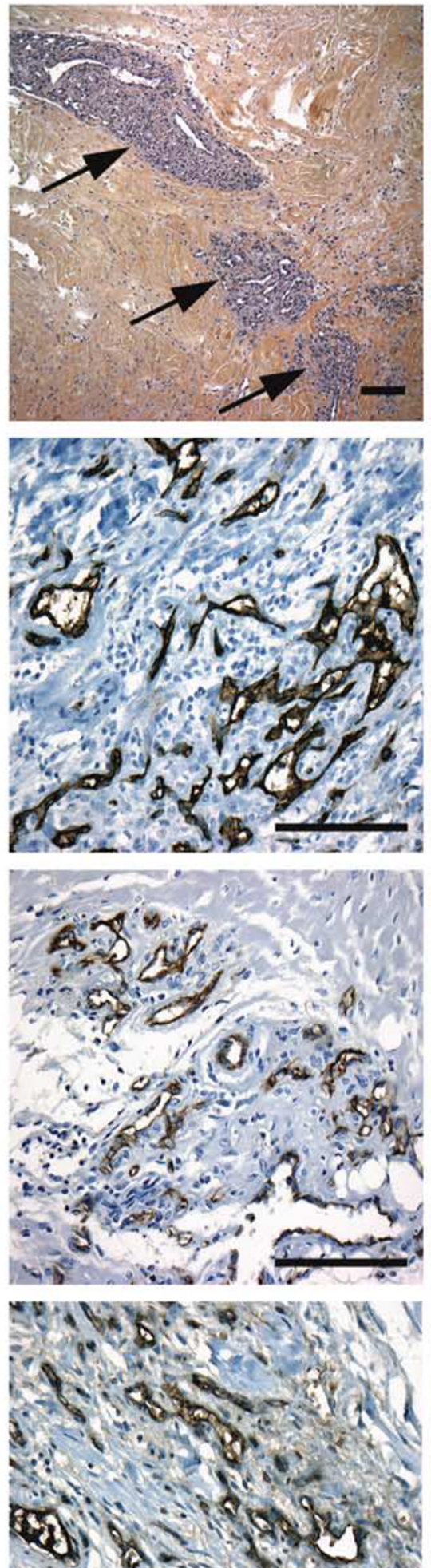

Nivi +2 s.

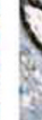

Patient 4
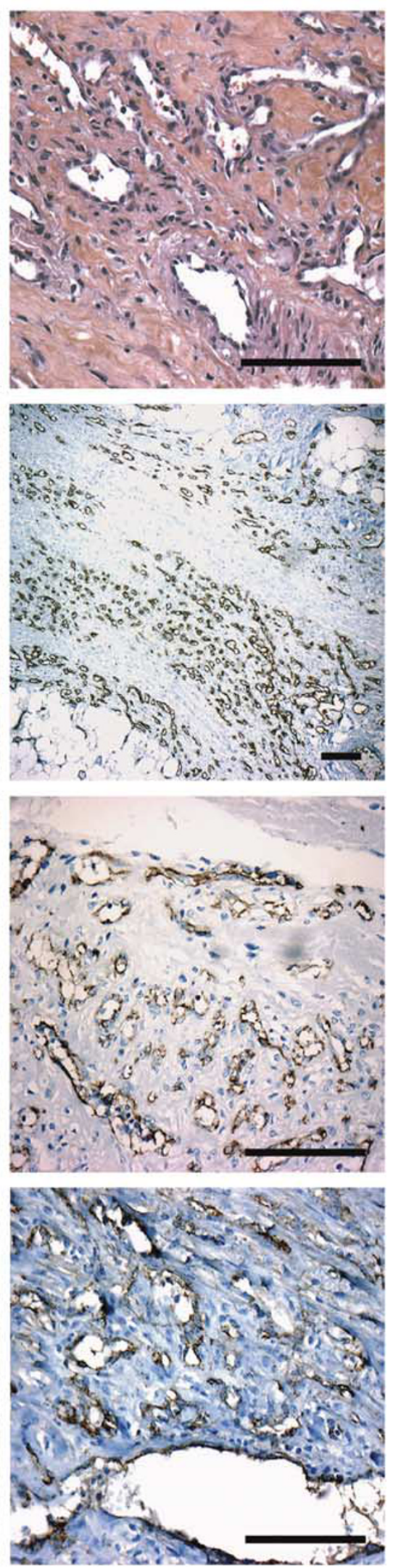

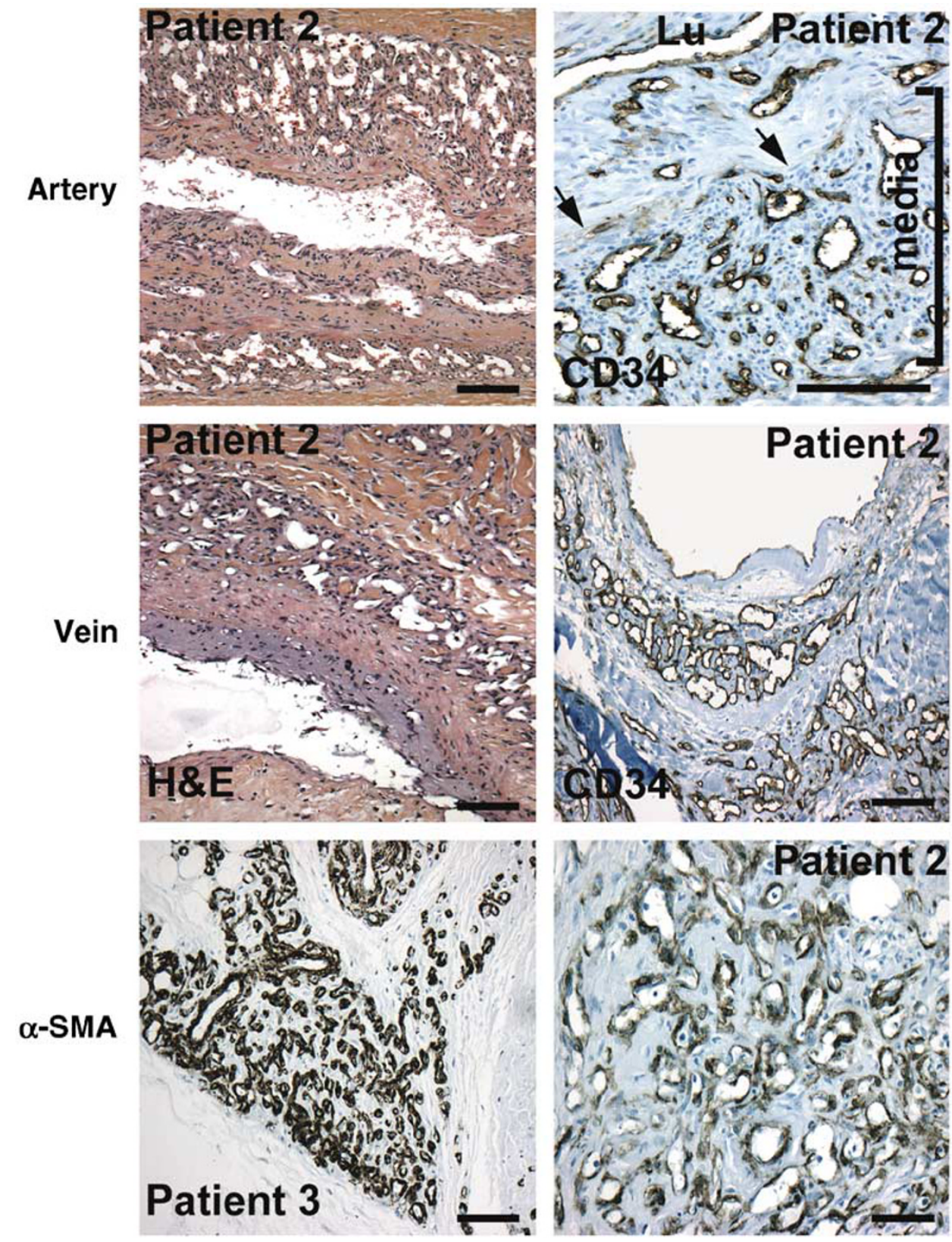

Figure 2 Angiogenesis in vessel walls and venule formation. (H\&E stain and CD34, and $\alpha$-smooth muscle actin ( $\alpha$-SMA) immunohistochemistry. Scale bar $=100 \mu \mathrm{m})$. Artery: H\&E: marked angiogenesis within the wall of a patent medium-sized artery, resulting in a spongy media; CD34 labeling confirmed the presence of newly formed vessels within the media. Artery lumen (Lu); internal elastic lumina (arrows). Vein: prominent angiogenesis within the wall of a patent medium-sized vein. Alpha-smooth muscle actin: most of the newly formed vessels are venules and small veins with a relatively dilated lumen and a wall made of a single layer of smooth muscle cells.

ischemic tissues of both patients and paired controls (Table 2).

As shown in Table 3, the extent of angiogenesis within the amputation specimens was variable in the three cases. As previously mentioned, angiogenesis was only present in the distal part of the amputation specimens. Widespread vascular proliferation or limited clusters of angiogenesis were observed in one to two-third of the distal blocks. Overall, the percentage of the angiogenesis area ranged from 1.2 to 13.2 percent of the total area of the sections of all distal blocks.

\section{Discussion}

This study reports for the first time an active angiogenesis process in humans treated with bone marrow mononuclear cells for critical limb ischemia. This angiogenesis was clearly related to the cell 

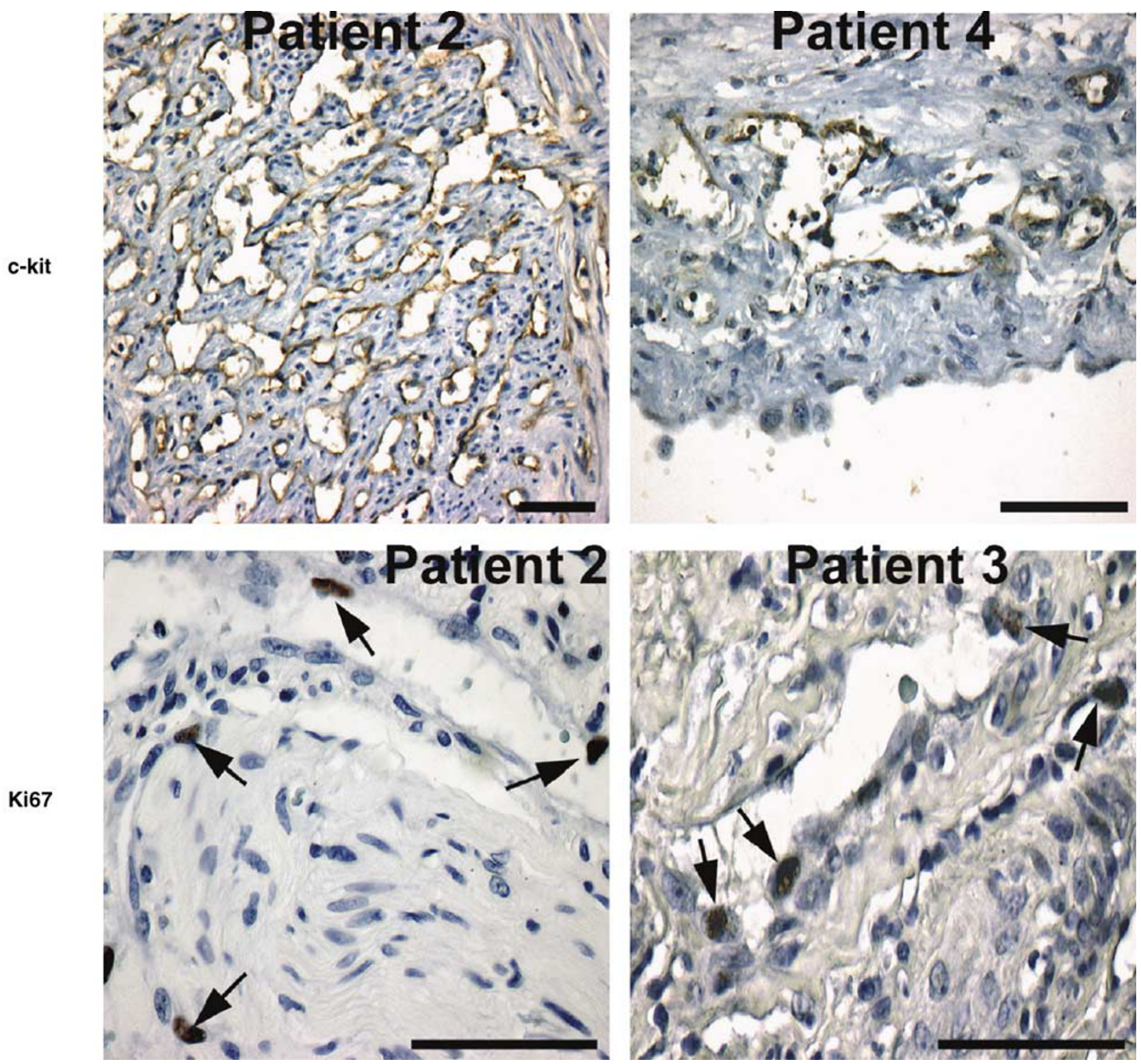

Figure 3 Active proliferation of endothelial cells. (c-kit and Ki67 immunohistochemistry. Scale bar $=50 \mu \mathrm{m}$ ). $c$-kit: strong expression of ckit in most of the newly formed vessels in patient 2; focal expression in patient 4. Ki67: Labeling of endothelial cell nuclei (arrows) demonstrates cell proliferation.

Table 2 Vascular density in angiogenic/non-angiogenic areas in patients and in ischemic tissues in paired controls

Chalkley count

\begin{tabular}{|c|c|c|c|}
\hline \multirow[b]{2}{*}{ Patient number } & \multicolumn{2}{|c|}{ Patient } & \multirow{2}{*}{$\frac{\text { Paired control }}{\text { In ischemic tissues }}$} \\
\hline & In angiogenic areas & In areas without angiogenesis & \\
\hline 2 & 15.6 & 4.8 & 3.4 \\
\hline 3 & 9.8 & 2.8 & 3.4 \\
\hline 4 & 9.8 & 3.6 & 3.2 \\
\hline Mean \pm s.e.m. & $11.8 \pm 1.9^{\mathrm{a}, \mathrm{b}}$ & $3.7 \pm 0.6^{\mathrm{c}}$ & $3.3 \pm 0.1$ \\
\hline
\end{tabular}

The individual Chalkley count is the mean of five measurements in CD34 stained sections with a 25 dots Chalkley graticule (unpaired $t$-test).

${ }^{\mathrm{a}}$ Patients in angiogenic areas vs controls in ischemic tissues, $P=0.01$.

${ }^{\mathrm{b}}$ Patients in angiogenic areas vs patients in areas without angiogenesis, $P=0.03$.

${ }^{\mathrm{c}}$ Patients in areas without angiogenesis vs controls in ischemic tissues, $P=0.53$ (paired $t$-test). 
Table 3 Extent of the angiogenic process within the amputation specimens

\begin{tabular}{|c|c|c|c|c|c|}
\hline \multirow[t]{2}{*}{ Patient } & \multicolumn{2}{|c|}{$\begin{array}{l}\text { Number of blocks with } \\
\text { angiogenesis/total number of blocks }\end{array}$} & \multicolumn{3}{|c|}{ Area with angiogenesis (distal blocks) } \\
\hline & Proximal & Distal & $\begin{array}{l}\text { Total angiogenesis } \\
\quad \text { area }\left(\mathrm{mm}^{2}\right)\end{array}$ & $\begin{array}{l}\text { Total section } \\
\text { area }\left(\mathrm{mm}^{2}\right)\end{array}$ & $\begin{array}{l}\text { Percentage of the } \\
\text { angiogenesis area }\end{array}$ \\
\hline 1 & NA & $0 / 3$ & NA & NA & NA \\
\hline 2 & $0 / 13$ & $9 / 12$ & 398 & 3024 & $13.2 \%$ \\
\hline 3 & $0 / 12$ & $4 / 13$ & 38 & 3016 & $1.2 \%$ \\
\hline 4 & NA & $9 / 20$ & 125 & 5619 & $2.2 \%$ \\
\hline
\end{tabular}

Distal, toe and forefoot; proximal, above the transmetatarsal section; NA, not assessed.
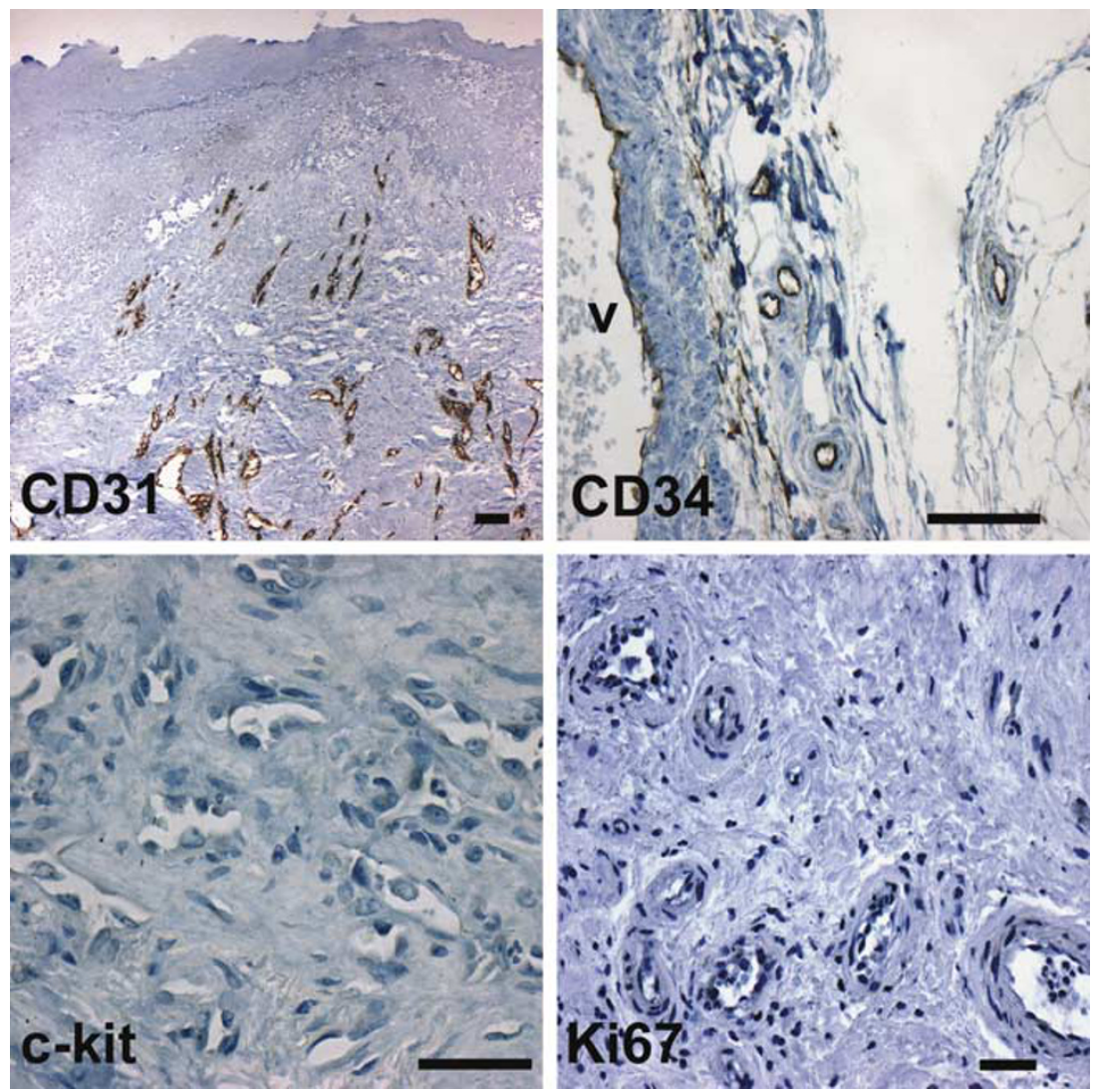

Figure 4 Control group. (CD31 and CD34 immunohistochemistry, scale bar $=100 \mu \mathrm{m}$; c-kit and Ki67 immunohistochemistry, scale $\mathrm{bar}=50 \mu \mathrm{m}$ ). CD31: normal vascular density in the dermis reflecting the absence of spontaneous angiogenesis in this ischemic area adjacent to gangrene $\left({ }^{*}\right)$. CD34: no angiogenesis is observed within the wall of the vein (v). Normal endothelial layer (arrows). $c$-kit: absence of c-kit expression in the endothelial cells of dermis vessels adjacent to an ischemic area. Ki67: Absence of proliferation of the endothelial cells in vessels adjacent to an ischemic area.

therapy. Because the study design of the OPTIPEC clinical trial did not allowed us any randomization between patients treated by cell therapy and patients whose amputated legs were analyzed as controls, we therefore used age- and sex-matched control patients with critical limb ischemia, who were amputated during the same period of time. Herein, we did not find any angiogenic process in these control samples. Furthermore, during the last 20 years, using the same dissection procedure on more than 400 amputation specimens, we have never observed such an angiogenic process.
The new microcirculation was functional, as shown by the presence of circulating blood cells in the vessel lumen. This neovascularization extended within the aponeurosis of the muscle and subcutaneous tissues. All the newly formed vessels were lined by a continuous endothelium expressing the endothelial cell markers CD31, CD34 and von Willebrand factor. Immunolabelling was negative for podoplanin, a glomerular podocyte membrane mucoprotein and a lymphatic endothelium marker that reliably distinguished lymphatic vessels from blood vessels. ${ }^{21}$ Positivity for Ki-67, a nuclear protein associated with 
cell proliferation, and c-kit, a tyrosine kinase receptor that normally controls the function of primitive hematopoietic cells, demonstrates active proliferation of endothelial cells within new vessels. ${ }^{22}$ Interestingly, this active proliferative process was observed in patients 3 and 4, respectively 12 and 8 weeks after cell injection.

However, this does not in itself demonstrate that the observed angiogenesis resulted from the incorporation of engrafted bone marrow mononuclear cells within the new vasculature, ie, a vasculogenic process. The ability of injected cells to be directly incorporated into a newly formed vasculature is still a matter of debate. ${ }^{23} \mathrm{~A}$ recent study using four different experimental models neatly demonstrated that mononuclear cells of bone marrow origin are not incorporated into vascular endothelial growth factorinduced blood vessels in mice, whereas marked recruitment of bone marrow-derived cells was seen at the sites of neoangiogenesis. ${ }^{24}$ These perivascular cells could play a supportive role or exert a paracrine action and thereby boost the vascular response to tissue ischemia. Likewise, there is no direct evidence that engrafted bone marrow mononuclear cells are incorporated during human angiogenesis. In our samples, the positivity of endothelial cells for both c-kit and Ki-67 suggested an active angiogenic process involving either engrafted cells or recruited progenitor endothelial cells undergoing local vasculogenesis. Direct proof of the involvement of injected cells in this process would require ex vivo labeling, but this is difficult to reconcile with good medical practice requirements.

Interestingly, no marked angiogenesis was seen at the injection site in the gastrocnemius muscle, but active angiogenesis was observed distally in the limb, mainly within the ischemic intermetatarsal spaces of the foot, in keeping with the fact that angiogenesis is mainly stimulated within the tissues with reactive increased $\mathrm{HIF}-1 \alpha$ in response to hypoxia. ${ }^{6}$ Cytokines that are released in response to hypoxia, such as VEGF and angiopoietin, are known to recruit progenitor cells. ${ }^{25,26}$ This may explain why most of the results of gene or cell therapies to stimulate angiogenesis in patients with intermittent claudication were negative, ${ }^{27,28}$ contrary to critical limb ischemia. Experimental VEGF gene therapy induced angiomatous lesions. ${ }^{29}$ In the first report of gene therapy with a plasmid encoding VEGF, Isner et $a l^{30}$ reported the occurrence of spider angiomas in the forefoot that spontaneously regressed 8 weeks after gene transfer. Biopsy and immunostaining of one of the angiomas also demonstrated extensive endothelial proliferation distal to the site of gene transfer. The authors suggested that the angiomatous lesions were limited to the distal part of the limb because of local upregulation of endothelial VEGF receptors. ${ }^{30}$ In the present report, widespread angiogenesis was also seen, mainly in patients 2 and 3 and may result from the sustained presence of angiogenic stimuli. However, histologi- cal (absence of cellular atypia and of abnormal mitosis) and immunohistochemical results (Glut-1 negativity) ruled out vascular tumorigenesis including angiosarcoma.

Finally, angiogenesis predominated within the walls of small and medium-sized patent distal arteries and veins, and in the surrounding soft tissues. This suggests that new vessels sprout from pre-existing vessels, and reflects a process of angiogenesis whatever the origin of the involved endothelial cells is. A recent report, using the same protocol of cell therapy, showed a few vasa-vasorum budding from parent vessels that expressed c-kit, within the gastrocnemius. ${ }^{31}$ Unfortunately, in this study, the distal and ischemic part of the limbs were not analyzed.

Our results therefore demonstrate for the first time in human that the treatment of critical limb ischemia with bone marrow-derived mononuclear cells can induce active and durable neoangiogenesis in the ischemic and distal part of the treated limb, although this may not prevent amputation in some patients with very severe ischemia. The fact that proliferation lasted more than 2 months after cell injection suggests that this therapeutic approach could trigger a self-sustained angiogenic response in this propitious ischemic environment. It remains to be shown whether this involves angiogenesis driven by a paracrine effect of injected cells and/or vasculogenesis in which the injected cells are incorporated into the new vasculature. ${ }^{32}$

\section{Acknowledgements}

We thank Isabelle Martinez and Marjorie Starck for their help with patient management. This study was supported by grants from PHRC (03-034), INSERM U765, ATC INSERM network and CIC Biothérapiehôpital Necker-Enfants Malades.

\section{Disclosure/Conflicts of interest}

None.

\section{References}

1 Hirsch AT, Haskal ZJ, Hertzer NR, et al. ACC/AHA 2005 Practice Guidelines for the management of patients with peripheral arterial disease (lower extremity, renal, mesenteric, and abdominal aortic): a collaborative report from the American Association for Vascular Surgery/Society for Vascular Surgery, Society for Cardiovascular Angiography and Interventions, Society for Vascular Medicine and Biology, Society of Interventional Radiology, and the ACC/ AHA Task Force on Practice Guidelines (Writing Committee to Develop Guidelines for the Management of Patients With Peripheral Arterial Disease): endorsed by the American Association of Cardiovascular and Pulmonary Rehabilitation; National Heart, Lung, and 
Blood Institute; Society for Vascular Nursing; TransAtlantic Inter-Society Consensus; and Vascular Disease Foundation. Circulation 2006;113:e463-e654.

2 Opie SR, Dib N. Local endovascular delivery, gene therapy, and cell transplantation for peripheral arterial disease. J Endovasc Ther 2004;11(Suppl 2):II151-II162.

3 Emmerich J. Current state and perspective on medical treatment of critical leg ischemia: gene and cell therapy. Int J Low Extrem Wounds 2005;4:234-241.

4 Carmeliet P. Angiogenesis in health and disease. Nat Med 2003;9:653-660.

5 Carmeliet P. Mechanisms of angiogenesis and arteriogenesis. Nat Med 2000;6:389-395.

6 Ho TK, Rajkumar V, Ponticos M, et al. Increased endogenous angiogenic response and hypoxia-inducible factor-1alpha in human critical limb ischemia. J Vasc Surg 2006;43:125-133.

7 Losordo DW, Dimmeler S. Therapeutic angiogenesis and vasculogenesis for ischemic disease. Part I: angiogenic cytokines. Circulation 2004;109:2487-2491.

8 Losordo DW, Dimmeler S. Therapeutic angiogenesis and vasculogenesis for ischemic disease: part II: cellbased therapies. Circulation 2004;109:2692-2697.

9 Asahara T, Murohara T, Sullivan A, et al. Isolation of putative progenitor endothelial cells for angiogenesis. Science 1997;275:964-967.

10 Gulati R, Jevremovic D, Peterson TE, et al. Diverse origin and function of cells with endothelial phenotype obtained from adult human blood. Circ Res 2003;93:1023-1025.

11 Yoon CH, Hur J, Park KW, et al. Synergistic neovascularization by mixed transplantation of early endothelial progenitor cells and late outgrowth endothelial cells: the role of angiogenic cytokines and matrix metalloproteinases. Circulation 2005;112:1618-1627.

12 Tateishi-Yuyama E, Matsubara H, Murohara T, et al. Therapeutic angiogenesis for patients with limb ischaemia by autologous transplantation of bone marrow cells: a pilot study and a randomised controlled trial. Lancet 2002;360:427-435.

13 Higashi Y, Kimura M, Hara K, et al. Autologous bone marrow mononuclear cell implantation improves endothelium-dependent vasodilation in patients with limb ischemia. Circulation 2004;109:1215-1218.

14 Saigawa T, Kato K, Ozawa T, et al. Clinical application of bone marrow implantation in patients with arteriosclerosis obliterans, and the association between efficacy and the number of implanted bone marrow cells. Circ J 2004;68:1189-1193.

15 Huang PP, Li SZ, Han MZ, et al. Autologous transplantation of peripheral blood stem cells as an effective therapeutic approach for severe arteriosclerosis obliterans of lower extremities. Thromb Haemost 2004;91:606-609.

16 Huang P, Li S, Han M, et al. Autologous transplantation of granulocyte colony-stimulating factor-mobilized peripheral blood mononuclear cells improves critical limb ischemia in diabetes. Diabetes Care 2005;28:2155-2160.

17 Beaujean F, Hartmann O, Kuentz M, et al. A simple, efficient washing procedure for cryopreserved human hematopoietic stem cells prior to reinfusion. Bone Marrow Transplant 1991;8:291-294.
18 Preti RA, Ahmed T, Ayello J, et al. Hemopoietic stem cell processing: comparison of progenitor cell recovery using the Cobe 2991 cell washer and the Haemonetics V50 apheresis system. Bone Marrow Transplant 1992;9:377-381.

19 Van Huyen JP, Batisse D, Heudes D, et al. Alteration of cytochrome oxidase subunit I labeling is associated with severe mitochondriopathy in NRTI-related hepatotoxicity in HIV patients. Mod Pathol 2006;19: 1277-1288.

20 Hansen S, Graban DA, Sorensen FB, et al. The prognostic value of angiogenesis by Chalkley counting in a confirmatory study design on 836 breast cancer patients. Clin Cancer Res 2000;6:139-146.

21 Al-Rawi MA, Mansel RE, Jiang WG. Molecular and cellular mechanisms of lymphangiogenesis. Eur J Surg Oncol 2005;31:117-121.

22 Heissig B, Werb Z, Rafii S, et al. Role of c-kit/Kit ligand signaling in regulating vasculogenesis. Thromb Haemost 2003;90:570-576.

23 Kinnaird T, Stabile E, Burnett MS, et al. Bone-marrowderived cells for enhancing collateral development: mechanisms, animal data, and initial clinical experiences. Circ Res 2004;95:354-363.

24 Zentilin L, Tafuro S, Zacchigna S, et al. Bone marrow mononuclear cells are recruited to the sites of VEGFinduced neovascularization but are not incorporated into the newly formed vessels. Blood 2006;107:35463554.

25 Asahara T, Takahashi $\mathrm{T}$, Masuda $\mathrm{H}$, et al. VEGF contributes to postnatal neovascularization by mobilizing bone marrow-derived endothelial progenitor cells. EMBO J 1999;18:3964-3972.

26 Smadja DM, Laurendeau I, Avignon C, et al. The angiopoietin pathway is modulated by PAR-1 activation on human endothelial progenitor cells. J Thromb Haemost 2006;2051-2058.

27 Rajagopalan S, Mohler III ER, Lederman RJ, et al. Regional angiogenesis with vascular endothelial growth factor in peripheral arterial disease: a phase II randomized, double-blind, controlled study of adenoviral delivery of vascular endothelial growth factor 121 in patients with disabling intermittent claudication. Circulation 2003;108:1933-1938.

28 Makinen K, Manninen H, Hedman M, et al. Increased vascularity detected by digital subtraction angiography after VEGF gene transfer to human lower limb artery: a randomized, placebo-controlled, double-blinded phase II study. Mol Ther 2002;6:127-133.

29 Lee RJ, Springer ML, Blanco-Bose WE, et al. VEGF gene delivery to myocardium: deleterious effects of unregulated expression. Circulation 2000;102:898-901.

30 Isner JM, Pieczek A, Schainfeld R, et al. Clinical evidence of angiogenesis after arterial gene transfer of phVEGF165 in patient with ischaemic limb. Lancet 1996;348:370-374.

31 Pignon B, Sevestre MA, Chatelain D, et al. Histological changes after implantation of autologous bone marrow mononuclear cells for critical leg ischemia. Bone Marrow Transplant 2007;39:647-648.

32 Sainz J, Sata M. Maintenance of vascular homeostasis by bone marrow-derived cells. Arterioscler Thromb Vasc Biol 2006;26:1196-1197. 